\title{
Evaluation of Two-Channel-Based Sound Source Localization using 3D Moving Sound Creation Tool
}

\author{
Hyun-Don Kim, Kazunori Komatani, Tetsuya Ogata, and Hiroshi G. Okuno \\ Department of Intelligence Science and Technology, \\ Graduate School of Informatics, Kyoto University, \\ Yoshida-honmachi, Sakyo-ku, Kyoto, 606-8501, Japan \\ \{hyundon, komatani, ogata, okuno\}@kuis.kyoto-u.ac.jp
}

\begin{abstract}
We proposed the way that can repeatedly evaluate the localization methods for moving sounds in the same condition regardless of a kind of methods and a number of microphones. And, we developed twochannel-based sound source localization integrated with a cross-power spectrum phase (CSP) analysis and EM algorithm. This one can localize several moving sounds and reduce localization error. Many sound source localization methods have already been developed. However, they could not be evaluated for moving sound in the same condition because it is hard to build database for moving sounds with accurate track information whenever making experiments. Also, to localize several moving sounds, conventional methods need a lot of microphone and/or prior information such as impulse response data. In this paper, we evaluated our sound localization method using $3 D$ moving sound creation tool and confirmed that our method with two microphones can well localize the voices of a moving talker without impulse response data.
\end{abstract}

\section{Introduction}

Sound Source Localization has been applied to such applications as sound source separation and speech enhancement. Recently, it generally has a tendency to be applied to robots for effective human robot interaction. Therefore, many methods of sound source localization for a humanoid robot have been developed and their performance has been generally improved.

However, although there are various sound source localization methods for the robot, those still have the problem that should be developed or improved:

1. There is no way to repeatedly evaluate the sound source localization for moving sounds in the same condition regardless of a kind of methods and a number of microphones.

2. Since it is natural that robots move and rotate their bodies and heads in order to track someone, the method should localize moving sounds while coping well with the effects created by moving microphones.

3. A lot of microphones and/or prior information such as impulse response data have the restriction in application to the robot.

In this paper, to evaluate moving sounds, we used 3D moving sound creation tool called SoundLocus Lite developed by arinis sound technologies (http:// www.arns.com/tech5.html). Therefore, we created moving sounds including accurate track information such as an azimuth and a distance according to a created frame or time. We then evaluated our sound localization method using created moving sounds instead of using sounds that we recorded while practically moving a speaker. This way enables us to evaluate various sound localization applications in the same condition.

In addition, we use cross-power spectrum phase (CSP) analysis [1] of sound signals obtained by only two microphones to localize the sound source and an expectation-maximization (EM) algorithm [2] is used to localize several sound sources and reduce localization errors. The system using this method can localize several moving sound sources by using only two microphones without the need for impulse response data. Therefore, this method can be applied to various types of systems as long as the delay of arrival (DOA) between the two microphones is known.

\section{Sound source localization}

For sound source localization, the latest systems for robots mostly use one of three methods: head-related transfer function (HRTF) [3, 4], multiple signal 
classification (MUSIC) [5, 6], and CSP [1, 7]. HRTF and MUSIC typically need impulse response data and an array of microphones in order to localize several sound sources. Impulse response data must thus be measured for every discrete azimuth and/or elevation before these methods can be applied to robots. Even though a lot of microphones and impulse response data would improve localization performance, they would also increase the calculation time. Furthermore, configuring the microphones in the robot would be problematic. On the other hand, CSP does not need impulse response data, and can accurately find the direction of a sound by only using two microphones. Also, to localize several sound sources and reduce localization errors, we developed a new method of estimating the number and localization of sound sources based on probability using an expectationmaximization (EM) algorithm [2].

\subsection{Cross-power spectrum phase analysis}

The direction of a sound source can be obtained by estimating the time delay of arrival (TDOA) between two microphones. When there is a single sound source, the TDOA can be estimated by finding the maximum value of the cross-power spectrum phase (CSP) coefficients [1] derived by

$$
\begin{gathered}
\operatorname{csp}_{i j}(k)=\operatorname{IFFT}\left[\frac{F F T\left[s_{i}(n)\right] F F T\left[s_{j}(n)\right]^{*}}{\left|F F T\left[s_{i}(n)\right]\right|\left|F F T\left[s_{j}(n)\right]\right|}\right] \\
\tau=\arg \max \left(\operatorname{csp}_{i j}(k)\right)
\end{gathered}
$$

where $k$ and $n$ are the number of samplings for the delay of arrival between two microphones, $s_{i}(n)$ and $s_{j}(n)$ are signals entering into the microphone $i$ and $j$ respectively, FFT (or IFFT) is the fast Fourier transform (or inverse FFT), * is the complex conjugate, and $\tau$ is the estimated TDOA. The sound source direction is derived by

$$
\theta=\cos ^{-1}\left(\frac{v \cdot \tau}{d_{\max } \cdot F_{s}}\right)
$$

where $\theta$ is the sound direction, $v$ is the sound propagation speed, $F_{s}$ is the sampling frequency, and $d_{\max }$ is the distance with the maximum time delay between two microphones. The sampling frequency of our system was $16 \mathrm{kHz}$.

\subsection{Localization of moving sounds using EM}

Figure 1 (A) shows the sound source localization events extracted by CSP according to time or frame lapses. We can see events that lasted $192 \mathrm{~ms}$ are used to train the EM algorithm to estimate the number and localization of sound sources. We experimentally decided that the appropriate interval for the EM algorithm was $192 \mathrm{~ms}$. Figure 1 (B) shows the training process for the EM algorithm to estimate the distribution of sound source localization events. Figure 1 (C) shows that the EM training results indicate the refined localizations of sound sources by iterating processes (A) and (B) in the same way. The interval for EM training is shifted every $32 \mathrm{~ms}$.

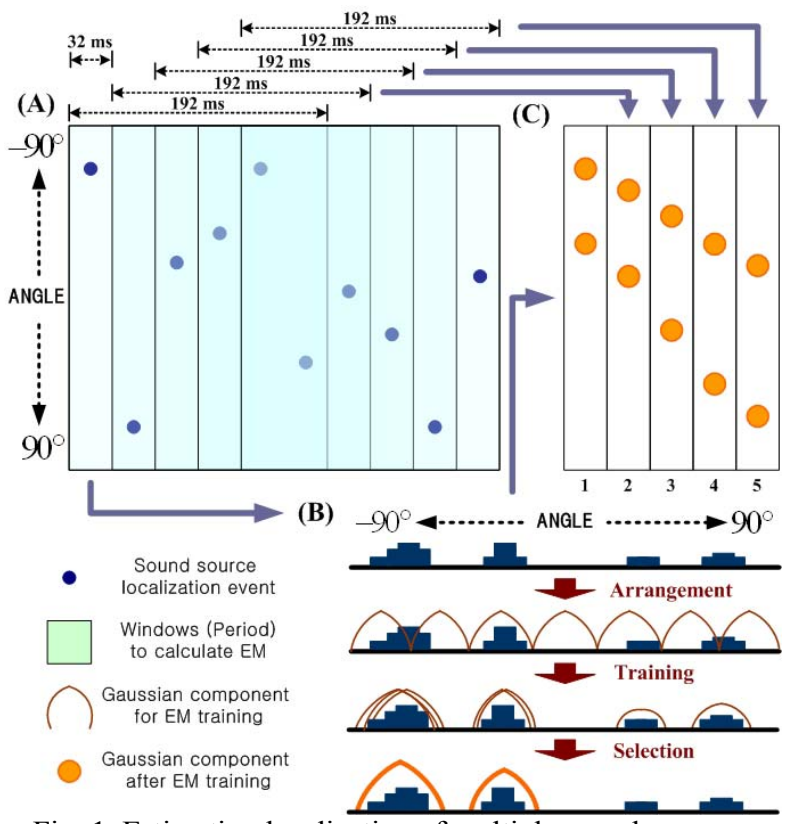

Fig. 1. Estimating localization of multiple sound sources.

Here, we explain the process of applying EM algorithm. Figure 2 describes the process in Figure 1 (B) in detail. In (A) of Figure 2, as the first step of EM training, sound source localization events were gathered for 192 ms. Next, Gaussian components defined by using equation (4) for training the EM algorithm were uniformly arranged on whole angles.

$$
P\left(X_{m} \mid \theta_{k}\right)=\frac{1}{\sqrt{2 \pi \sigma_{k}^{2}}} e^{-\frac{\left(X_{m}-\mu_{k}\right)^{2}}{2 \sigma_{k}{ }^{2}}}
$$

where $\mu_{k}$ is the mean, $\sigma^{2}{ }_{k}$ is the variance, $\theta_{k}$ is a parameter vector, $m$ is the number of data, and $k$ is the number of mixture components. At that time, in (A) of Figure 2, the $\mu$ and $\sigma$ parameters in Gaussian components are the respective center and radius values of each component. Then, the sound localization events are applied to the arranged Gaussian components to find the parameter vector, $\theta_{k}$, describing each component density, $P\left(X_{m} \mid \theta_{k}\right)$, through 
iterations of the E and M steps. This EM step is described as follows:

1) E-step: The expectation step essentially computes the expected values of the indicators, $P\left(\theta_{k} \mid X_{m}\right)$, where each sound source localization event $X_{m}$ is generated by component $k$. Given $N$ is the number of mixture components, the current parameter estimates $\theta_{k}$ and weight $w_{k}$, using Bayes' Rule derived as

$$
P\left(\theta_{k} \mid X_{m}\right)=\frac{P\left(X_{m} \mid \theta_{k}\right) \cdot w_{k}}{\sum_{k=1}^{N} P\left(X_{m} \mid \theta_{k}\right) \cdot w_{k}}
$$

2) M-step: At the maximization step, we can compute the cluster parameters that maximize the likelihood of the data assuming that the current data distribution is correct. As a result, we can obtain the recomputed mean using Equation (6), the recomputed variance using Equation (7), and the recomputed mixture proportions (weight) using Equation (8). The total number of data is indicated by $M$.

$$
\begin{gathered}
\mu_{k}=\frac{\sum_{m=1}^{M} P\left(\theta_{k} \mid X_{m}\right) X_{m}}{\sum_{m=1}^{M} P\left(\theta_{k} \mid X_{m}\right)} \\
\sigma_{k}^{2}=\frac{\sum_{m=1}^{M} P\left(\theta_{k} \mid X_{m}\right) \cdot\left(X_{m}-\mu_{k}\right)^{2}}{\sum_{m=1}^{M} P\left(\theta_{k} \mid X_{m}\right)} \\
w_{k}=\frac{1}{N} \sum_{m=1}^{M} P\left(\theta_{k} \mid X_{m}\right)
\end{gathered}
$$

(A)

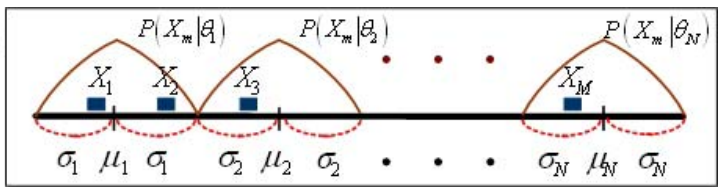

(B)

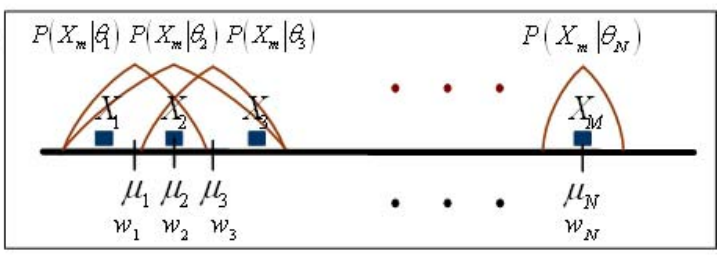

(C)

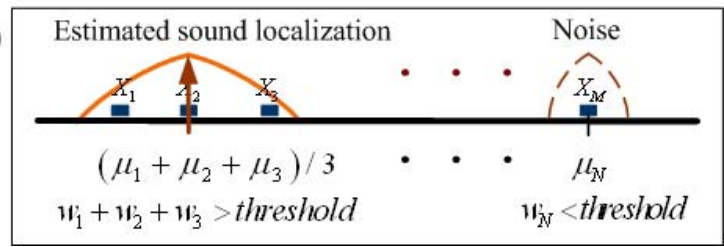

Fig. 2. Process of EM algorithm for estimating sound sources.

After the $\mathrm{E}$ and $\mathrm{M}$ steps are iterated an adequate number of times, the estimated mean, variance, and weight based on the current data distribution can be obtained. Then, in (B) of Figure 2, the weight and mean of Gaussian components are reallocated based on the density and distribution of the histogram data. Finally, in (C) of Figure 2, if the components overlap, each weight value of overlapping Gaussian components will be added. After that, if the weight value is higher than a threshold value, the system can determine the localization of the sound source by computing the average mean of the overlapping Gaussian components. In contrast, components with small weights are regarded as noise and will be removed.

\section{Experiments and results}

We created moving sounds using the 3D moving sound creation tool called SoundLocus Lite developed by arinis sound technologies (http://www.arns.com/ tech5. html). Figure 3 shows GUI for operating this tool. This tool can convert any wav file into twochannel-based moving sounds according to track of desire. Therefore, after we designated the value for the velocity of moving sounds beforehand, we can freely make moving sounds while operating a joystick.

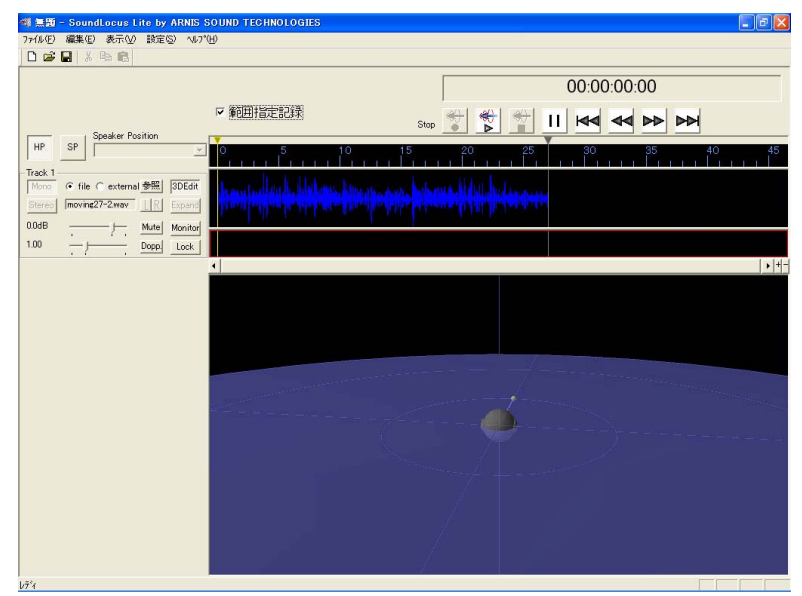

Fig. 3. GUI of 3D moving sound creation tool.

In this paper, to evaluate our method for moving sounds, we created six moving voice signals which were rotated from $1^{\circ}$ to $360^{\circ}$ with $1.0,1.5,2.0 \mathrm{~m} / \mathrm{s}$ at about $2.0 \mathrm{~m}$ and $4.0 \mathrm{~m}$ from the center. We then performed our sound localization using created moving sounds. The top of Figure 4 shows the experiment setup. We used two omnidirectional microphones installed at the left and right ear position of the humanoid robot SIG2 [7] and used two fixed speakers to emit created moving sounds at $0.5 \mathrm{~m}$ from left and 
right sides of microphones. The diamond shape in graphs of Figure 4 indicated the track of created moving sounds and the square shape indicated the results of localizing created moving sounds.
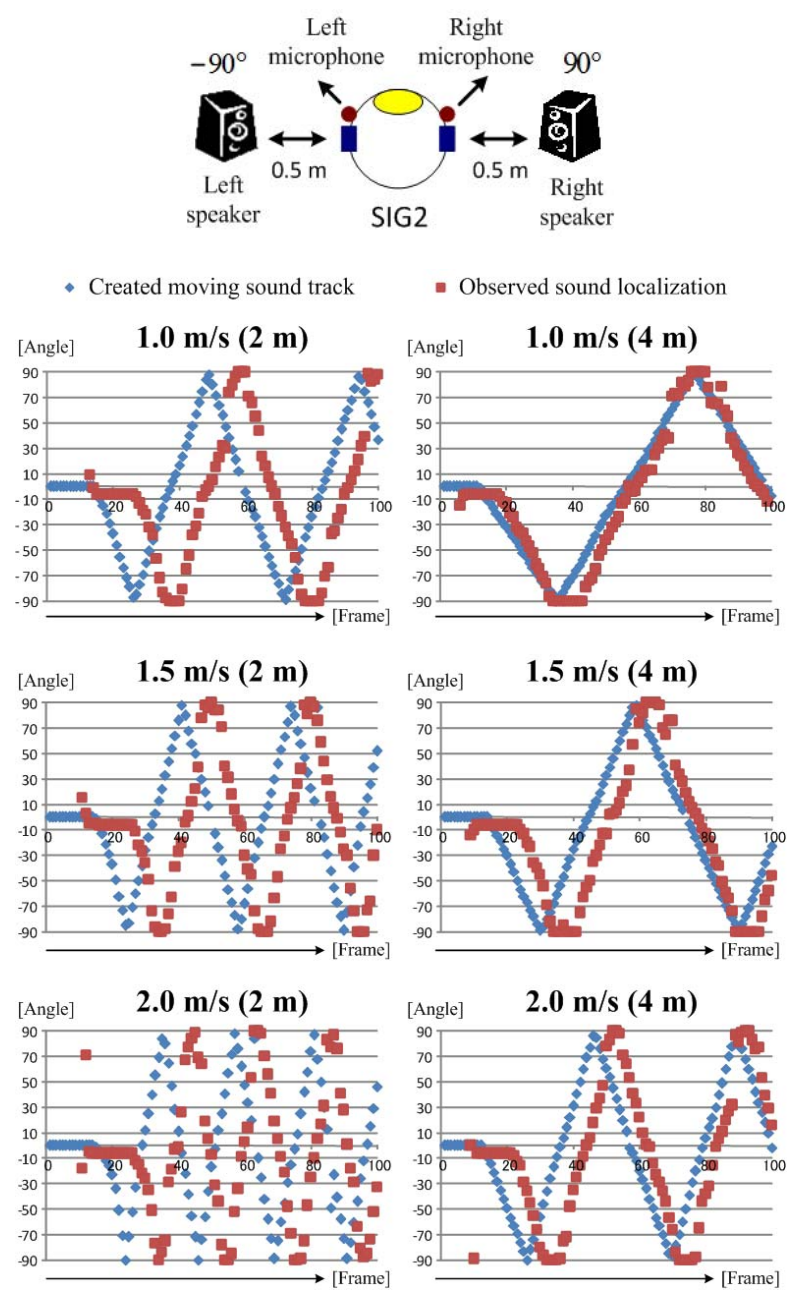

Fig. 4. Experimental setup and results of sound localization.

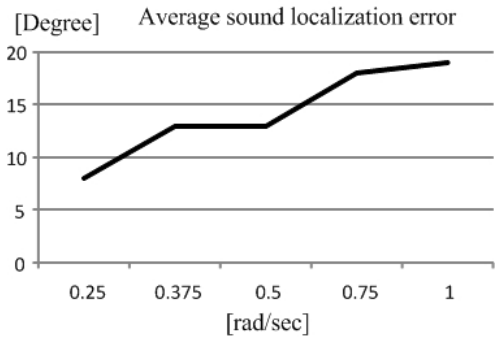

Fig. 5. Average sound localization error for our method.

Our method obtained the average sound localization error of $8^{\circ}$ and $13^{\circ}$ when an angular velocity of moving sounds was $0.25 \mathrm{rad} / \mathrm{sec}(1.0 \mathrm{~m} / \mathrm{s}$ at about $4 \mathrm{~m}$ distance $)$ and $0.5 \mathrm{rad} / \mathrm{sec}(1.0 \mathrm{~m} / \mathrm{s}$ at about $2 \mathrm{~m}$ distance) as shown in Figure 4 and 5. Since the average walking speed of healthy adults is $1.0 \mathrm{~m} / \mathrm{s}$, we can know that our method can cope well with the voices of a walking talker.

\section{Conclusion}

We proposed the way that can repeatedly evaluate the sound source localization in the same condition regardless of a kind of methods and a number of microphones. Also, we developed two-channel-based sound source localization integrated a cross-power spectrum phase (CSP) analysis and EM algorithm. Testing showed that our method can reliably locate the voice signals moving at less than $0.5 \mathrm{rad} / \mathrm{sec}$. In future work, we plan to develop the system that can distinguish whether sound signals are coming from the front or back by rotating a robot's head, in which two microphones are located. Also, we consider enabling our method to localize two moving sounds simultaneously.

\section{References}

[1] T. Nishiura, T. Yamada, S. Nakamura, and K. Shikano, "Localization of multiple sound sources based on a CSP analysis with a microphone array," IEEE/ICASSP Int. Conf. Acoustics, Speech, and Signal Processing, June (2000) pp. 1053-1056.

[2] T. K. Moon. "The Expectation-Maximization algorithm," IEEE Signal Processing Magazine, Nov. (1996) 13(6) pp. $47-$ 60.

[3] C. I. Cheng \& G. H. Wakefield, "Introduction to HeadRelated transfer Functions (HRTFs): Space," Journal of the Audio Engineering Society, vol. 49, no. 4, pp.231-248, 2001.

[4] S. Hwang, Y. Park, and Y. Park, "Sound Source Localization using HRTF database," in Proc. Int. Conf. on Control, Automation, and Systems (ICCAS2005), June, 2005, pp.751-755.

[5] R. O. Schmidt, "Multiple Emitter Location and Signals Parameter Estimation," IEEE Trans. Antennas Propag., AP34, 1986, 276-280.

[6] I. Hara, F. Asano, Y. Kawai, F. Kanehiro, and K. Yamamoto, "Robust speech interface based on audio and video information fusion for humanoid HRP-2," IEEE/RSJ International Conference on Intelligent Robots and Systems (IROS-2004), Oct. (2004) pp. 2404-2410.

[7] H. D. Kim, K. Komatani, T. Ogata, and H. G. Okuno, "Auditory and Visual Integration based Localization and Tracking of Human in Daily-life Environments," IEEE/RSJ International Conference on Intelligent Robots and Systems (IROS-2007), Nov. (2007) pp.2021-2027. 